\title{
Sociodemographic disparities in energy insecurity among low-income households before and during the COVID-19 pandemic
}

\author{
Trevor Memmott, Sanya Carley $₫$ $₫$, Michelle Graff and David M. Konisky $₫$
}

\begin{abstract}
Energy insecurity is a growing public health threat among low-income populations in the United States. Prior research has shown that energy insecurity is associated with adverse health effects and can lead people to engage in risky coping strategies. Here we evaluate rates of energy insecurity, which factors contribute to it, and how the early months of the COVID-19 pandemic exacerbated the problem. We show that energy insecurity is highly prevalent among households at or below $200 \%$ of the federal poverty line. We further show that Black and Hispanic households are more likely to experience energy insecurity and face utility disconnection, as are households with young children, individuals that require electronic medical devices and those in dwellings with inefficient or poor conditions. These conditions exist under normal circumstances, and the COVID-19 pandemic seems to have exacerbated the overall incidence of energy insecurity.
\end{abstract}

E nergy insecurity-conceptualized as the inability of a household to meet its basic energy needs ${ }^{1,2}$-is a pervasive problem in the United States and elsewhere ${ }^{3}$. Scholars use terms such as energy poverty, fuel poverty and energy vulnerability to capture similar conditions ${ }^{4-8}$ that involve both affordability and access to reliable energy sources. Although electricity is necessary for essential services (for example, heating, cooling, cooking, lighting and medical devices), policymakers have failed to recognize the scope and scale of the energy insecurity problem ${ }^{9}$. In response to energy insecurity, vulnerable households are more likely to engage in risky behaviours to meet their energy needs ${ }^{10}$, which include using high-interest payday loans ${ }^{11}$, a reliance on dangerous heating sources, such as space heaters or ovens ${ }^{12}$, or forgoing other basic needs, such as food and medical care ${ }^{13}$. Individuals in energy-insecure households are more likely to remain in poverty for longer periods of time ${ }^{14}$ and are more likely to suffer adverse mental and physical health consequences ${ }^{15-18}$, which include an increased incidences of death ${ }^{19}$; these impacts are especially prevalent for children ${ }^{20,21}$ and the elderly ${ }^{22,23}$.

The economic disruption thus far caused by the COVID-19 pandemic has increased pre-existing inequalities, which include those associated with energy insecurity. Black and Hispanic communities have been impacted by disproportionate rates of coronavirus-related infections, hospitalizations and mortality ${ }^{24,25}$; and Black and Hispanic populations, young workers, mothers of school-aged children, and low-educated individuals have experienced large employment shocks ${ }^{26-29}$. This study expands this work by examining which American families experience energy insecurity during a public health crisis. In so doing, we also contribute to a growing literature on energy justice, material hardship and vulnerable populations $\mathrm{s}^{1,30,31}$.

The extant literature on energy insecurity has found that Blacks, Hispanics, those without a college degree and households with young children are all more likely to be energy insecure ${ }^{2,31,32}$. These studies provide a strong foundation that scholars and practitioners currently rely on to understand the problem of domestic energy insecurity. Current knowledge of the scope and scale of energy insecurity, however, primarily comes from the analysis of two limited data sources: the Residential Energy Consumption Survey (RECS) ${ }^{33}$ and the American Community Survey (ACS). The RECS contains few energy insecurity questions, is administered only every four years, does not provide state-level geographical identifiers, and examines the public at large rather than the most vulnerable populations; and, although the ACS contains items on energy expenditures, it does not capture affordability ${ }^{34}$. Analyses using these datasets have made important contributions to our understanding of energy insecurity, but neither the ACS nor the RECS enable a comprehensive empirical analysis of US energy insecurity.

To address these shortcomings, we administered a nationally representative survey of 2,381 adults with household incomes at or below $200 \%$ of the Federal Poverty Level (FPL) in April and May 2020. The survey captures multiple measures of energy insecurity, collects demographic, health and housing conditions information, and enables the evaluation of energy insecurity over the course of a year, which allows us to compare the prevalence and correlates of energy insecurity during both 'normal' circumstances and in the early months of the COVID-19 pandemic ${ }^{35,36}$.

To summarize our findings, energy insecurity is highly prevalent among low-income American households, especially among households that identify as Black and Hispanic. We found that those who require use of an electronic medical device and live in poor or less-efficient housing conditions experience higher rates of energy insecurity. The COVID-19 pandemic has thus far deepened the prevalence of energy insecurity among low-income households, as some speculated may occur ${ }^{37}$, with some indication of growing disparities.

\section{Describing energy insecure households}

We evaluate energy insecurity across three measures, which represent a range in severity: inability to pay an energy bill, receipt of a utility disconnection notice and disconnection from energy service. All measures rely on respondent recall about conditions over the past year (from roughly April/May 2019 to April/May 2020) and over the past month (April/May 2020), respectively. Although we cannot rule out recall bias, we believe it is likely that most 


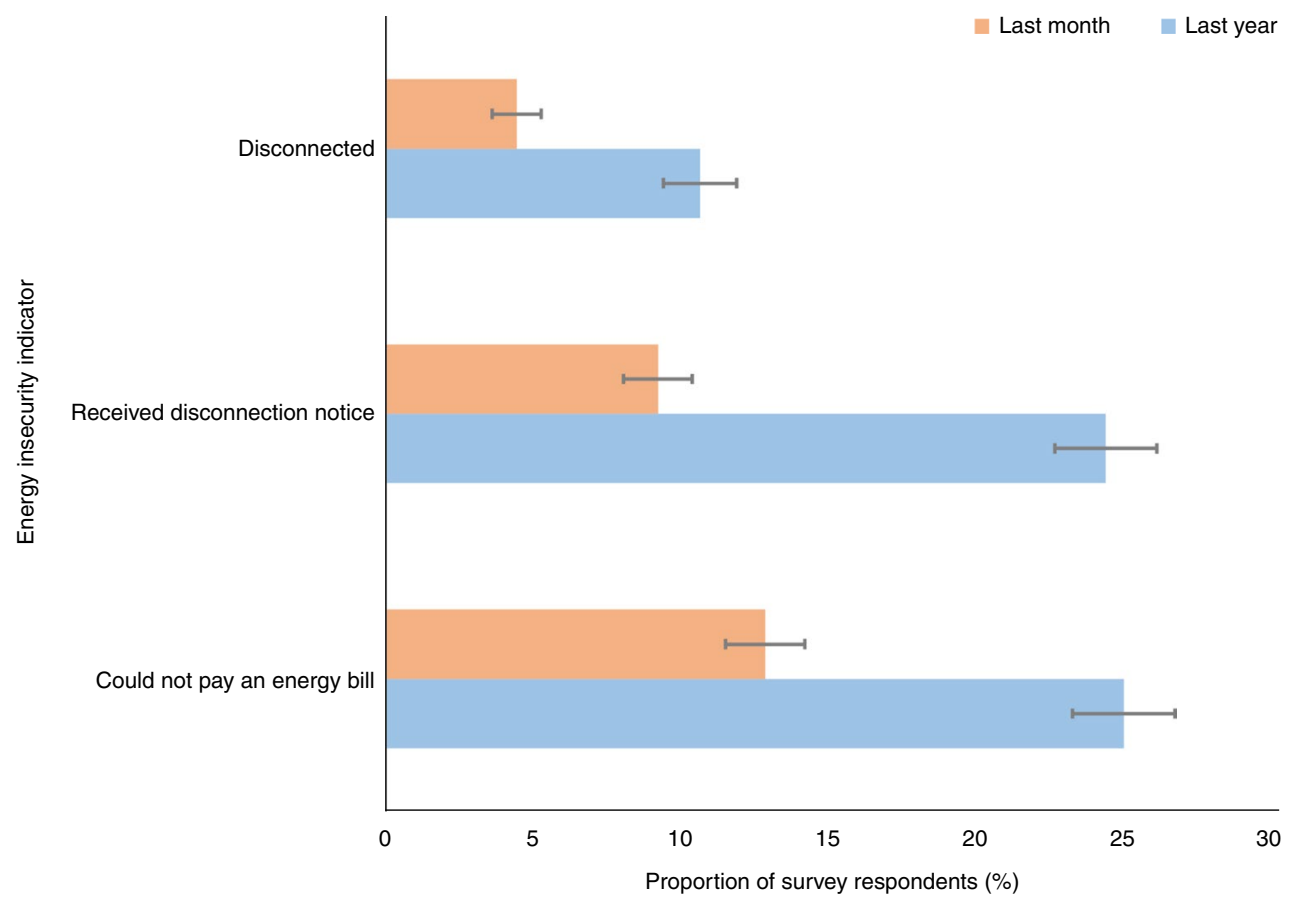

Fig. 1 | Proportion of survey respondents facing energy insecurity. Respondents who reported an inability to pay their energy bill, received a disconnection notice or were disconnected in the past year (blue) and in the past month (orange). The grey bars represent 95\% Cls $(n=2,381)$.

respondents accurately remember events such as being unable to pay a bill, learning that they will soon be disconnected unless urgent action is taken, or being disconnected from the grid and losing access to electricity.

The survey results (Fig. 1) reveal that $25 \%$ (the $95 \%$ confidence interval (CI) is $23.0-27.0 \%$ ) of low-income households could not afford to pay an energy bill in the past year and nearly $13 \%$ (95\% CI $10.9-14.9 \%$ ) could not afford their bill in the past month. In the past year, just over $25 \%$ (95\% CI 23.6-27.6\%) of respondents received a notice from their utility provider that their power may be disconnected due to lack of payment, and over $10 \%$ (95\% CI 8.6-12.6\%) had their service disconnected. In the past month, during the onset of the economic dislocation from the COVID-19 pandemic, 9\% (95\% CI $7.2-11.2 \%$ ) of respondents had received a notice and $4 \%$ (95\% CI 2.4-6.4\%) were disconnected.

To further characterize the general prevalence of energy insecurity among low-income US households, as well as its incidence since the onset of the COVID-19 pandemic, we combined the survey data with estimates from the 2018 ACS for those who live at or below $200 \%$ of the FPL to approximate the number of US households and individuals that are energy insecure. Specifically, we extrapolated from the survey data to the US population by multiplying the proportion of survey respondents that reported some level of energy insecurity by the number of US households (individuals) that the ACS estimates to be at or below $200 \%$ of the FPL. As shown in Table 1 , we estimate that, in the past year, approximately 4.7 (24.3) million households (individuals) could not pay an energy bill, and just over $50 \%-2.4$ (12.5) million households (individuals) - indicated that at least one of the bills they could not pay was in the past month. Although we estimate that 4.8 (24.9) million households (individuals) received a disconnection notice and approximately $41 \%$ of these households were disconnected in the past year, the proportion of households that were disconnected from the grid after they received a notice rose to about $48 \%$ during the COVID- 19 pandemic.

To put these estimates into context, we compared them with the RECS survey, which provides a nationally representative snapshot
Table 1 | Estimates of US households experiencing energy insecurity

Could not pay Received notice Disconnected
an energy bill

Number of households $\times 10^{6}$ (estimate range)

$\begin{array}{llll}\begin{array}{l}\text { Past year (April/ } \\ \text { May 2019 to }\end{array} & 4.7(4.4-5.2) & 4.8(4.5-5.3) & 2.0(1.6-2.4) \\ \begin{array}{l}\text { April/May 2020) } \\ \text { Past month }\end{array} & 2.4(2.1-2.8) & 1.7(1.4-2.1) & 0.8(0.5-1.2) \\ \begin{array}{l}\text { (April/May 2020) } \\ \text { Number of individuals } \times 10^{6} \text { (estimate range) }\end{array} & \\ \begin{array}{l}\text { Past year (April/ } \\ \text { May 2019 to }\end{array} & & & \\ \text { April/May 2020) } & & & \\ \begin{array}{l}\text { Past month } \\ \text { (April/May 2020) }\end{array} & 12.5(10.6-14.5) & 8.9(7.0-10.9) & 4.3(2.3-3-6.2)\end{array}$

The approximate number of households-defined by the ACS as family members who reside together-and individuals who suffer from energy insecurity in the United States based on the 2018 ACS estimates ${ }^{54}$ of households that live at or below $200 \%$ of the FPL and the proportion of respondents that reported they could not pay an energy bill, received a disconnection notice or were disconnected from energy service. The range represents the $95 \% \mathrm{Cls}$ for the estimates.

of all domestic housing units in the United States. According to the most recent RECS, 17 million households received a disconnection notice in 2015, and 3 million households had their electricity disconnected. We can approximate from our 2020 survey that roughly $30 \%$ of all US households who received disconnection notices had incomes at or below $200 \%$ of the FPL, and nearly two-thirds of those that were disconnected came from households at or below $200 \%$ of the FPL. Although these approximations should be interpreted cautiously given differences in survey methodology and timing, we can infer that low-income households are being disconnected at much 


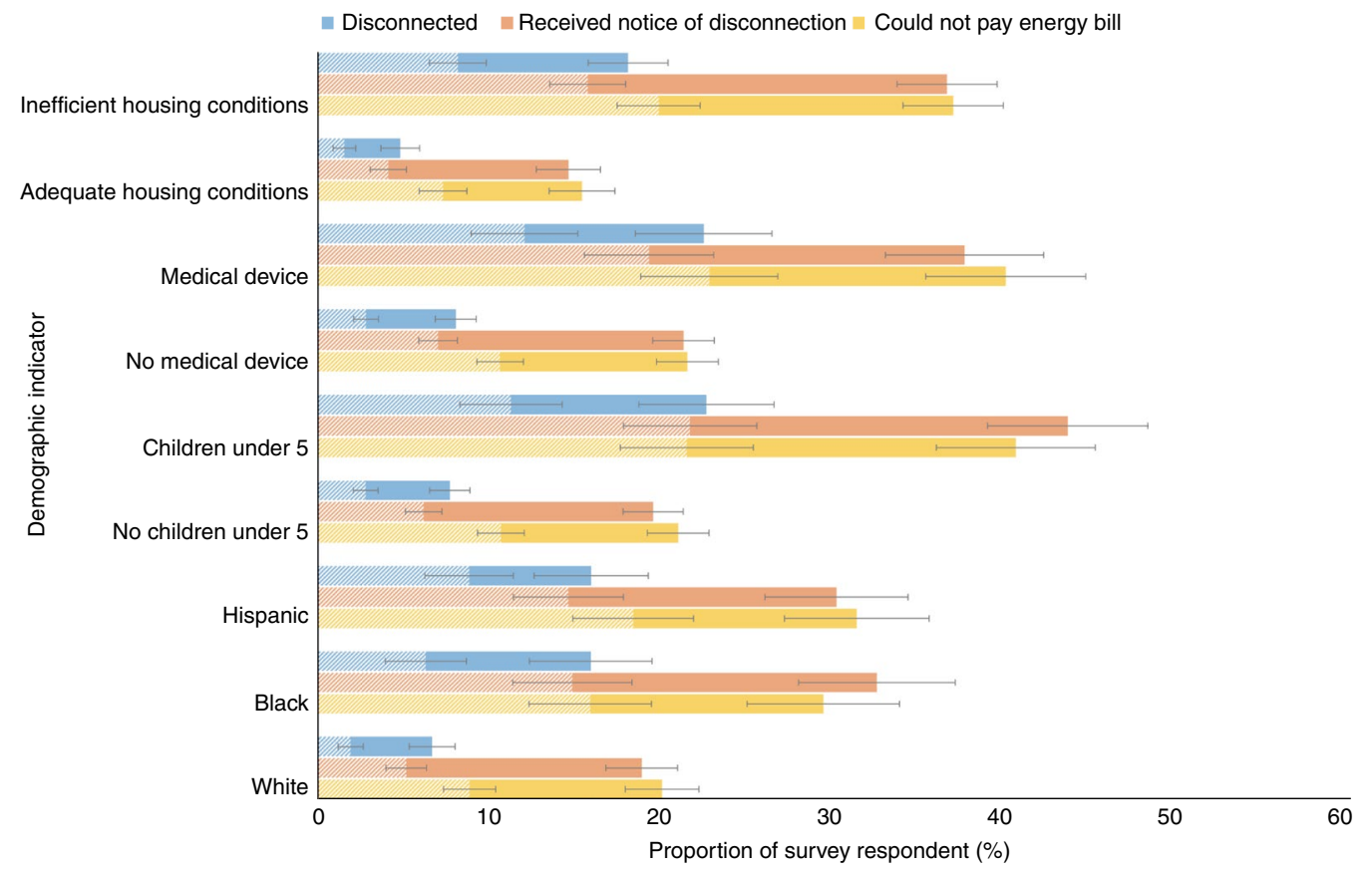

Fig. 2 | Energy insecurity by demographic characteristics. The proportion of households that could not afford an energy bill (yellow), received a disconnection notice (orange), or were disconnected from the grid (blue) in the past year (full bars) compared with those in the past month alone (dashed components), according to key indicators. Grey bars represent the $95 \% \mathrm{Cls}(n=2,381)$.

higher rates after receipt of a notice from their utility than averageto high-income households.

To further understand energy insecure households, we disaggregated the indicators of energy insecurity by race, number of dependents, health and housing conditions. These findings are illustrated in Fig. 2, in which each full bar represents the proportion of survey respondents who reported being energy insecure in the past year. We find that Black households, Hispanic households, households with young children, those with a member that relies on an electronic medical device and those who reside in poor housing conditions (for example, mould, holes in the wall and/or floor, plumbing problems, broken heating and air conditioning, exposed electrical sockets, non-working stove and/or refrigerator, or poor insulation) all reported higher incidences of energy insecurity. These characteristics are positively associated with all three energy insecurity measures, which demonstrates that these disparities are prevalent during typical circumstances (that is, the year prior to the onset of the COVID-19 pandemic, which was characterized by a normal-to-strong US domestic economy ${ }^{38}$ ).

Figure 2 further suggests that many of these disparities have grown since the beginning of the COVID-19 pandemic. Although the survey data do not enable us to make precise year-on-year comparisons, there was a higher prevalence of energy insecurity across many of these sociodemographic and household attributes during the early period of the COVID-19 pandemic (dashed bars in Fig. 2). For example, $20 \%$ of White households and $30 \%$ of Black households could not pay an energy bill in the past year, which accounts for 12 full months; whereas 9\% of White households and $16 \%$ of Black households indicated that they had trouble paying their energy bill in just the past month alone, a month that is not typically a weather aberration, nor was it extreme in 2020. Additionally, Hispanic respondents were 2.4 times more likely than White respondents to be disconnected from the grid in the past 12 months, but 4.7 times more likely to be disconnected during the early period of the COVID-19 pandemic. Similar patterns emerged across the other indicators displayed in Fig. 2, which provides some evidence of an increase in disparities in energy insecurity during the beginning of the public health and economic crisis that results from the COVID-19 pandemic.

\section{Sociodemographic predictors of energy insecurity}

To better identify the determinants of household energy insecurity, we estimated a series of logistic regression models. This analysis evaluated the correlates of energy insecurity over the past year and in the past month to compare patterns of household energy insecurity during typical circumstances and the potential unique hardships that these households experienced since the onset of the pandemic. For brevity, we present the results of our models in Fig. 3 for the demographic, health and housing characteristics that are consistently statistically associated with at least two of our indicators of energy insecurity. For clarity, we discuss our findings in terms of odds ratios. Full model regression results are also presented in Supplementary Table 1. In the models, we also include measures for households with a disabled member, other race categories (which include Asian, Native American and mixed households), whether the household is situated in an urban or rural zip code, whether the household received government assistance and the respondent's age, gender, employment status and level of educational attainment (see Supplementary Table 2 for a full list of variables).

As illustrated in Fig. 3, Black respondents were more likely than White respondents to be energy insecure across all three energy insecurity measures in the past year, with Hispanic households more likely to receive a notice of disconnection or be disconnected from the electric grid. More specifically, compared with White households, Black and Hispanic households experienced more severe forms of energy insecurity at higher rates. Black households experienced 1.9 times greater odds of receiving a disconnection notice and 2.2 times greater odds of having their utility service disconnected than the White household respondents. Hispanic households faced disconnection at 1.9 times greater odds than White household respondents.

Moreover, the COVID-19 pandemic appears to have increased racial disparities. The regression analysis suggests that Black 


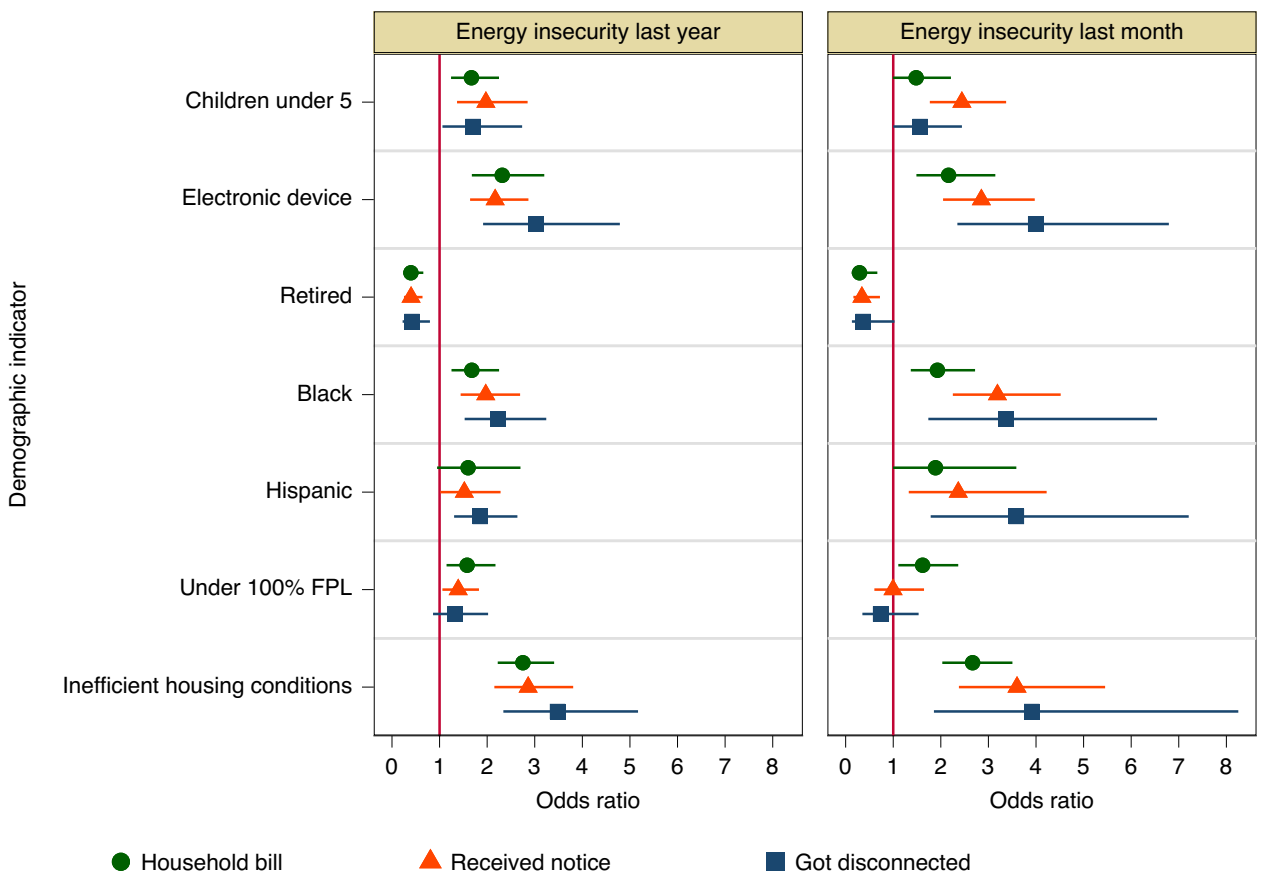

Fig. 3 | Sociodemographic correlates of energy insecurity. Values are odds ratios with $95 \% \mathrm{Cls}$, estimated from logistic regression models ( $n=2,359)$. The models also include variables for household member with a disability, unemployed, other race or ethnicity, gender, age, education, dwelling type (mobile home, apartment or attached home), percentage of urban area within the zip code and receipt of government assistance. The full model estimates are provided in Supplementary Table 1.

respondents were more likely to face all three forms of insecurity and Hispanic respondents were more likely to receive a notice or be disconnected in the time since the pandemic began compared to over the course of the previous year. Blacks (Hispanics) were at 3.4 (3.6) greater odds of being disconnected from their utility service during the pandemic than White respondents. These temporal differences in prevalence across racial groups suggest that energy insecurity intensified during the early months of the pandemic, although we cannot infer this definitively from the statistical analysis because the CIs for the coefficients across the models overlap.

Similarly, respondents who live in households in which at least one person requires the use of a home electronic medical device were more likely to be unable to pay their bills, to receive a notice and to be disconnected, both over the course of the prior year and during the early period of the COVID-19 pandemic. During the early months of the pandemic, for example, electronic device users faced 4.0 times greater odds of having their utility service disconnected compared with those who did not require the use of a medical device, which suggests that this vulnerable population is at high risk of losing power and facing tangible health risks. In addition, households with children under the age of five, those who live in poor or inefficient dwelling conditions, and those that have received government assistance in the past month also faced a higher risk of experiencing energy insecurity. We found that retired individuals, on average, were less energy insecure.

Lastly, respondents that had a household income under $100 \%$ of the FPL were more likely to be unable to pay their energy bill in both the past year and the past month as well as more likely to receive a disconnection notice in the past year, as compared with those whose household incomes were between 150 and $200 \%$ of the FPL. The lack of a statistical association between income levels and disconnection from the electric grid is a noteworthy null finding. It suggests that economic characteristics are not the sole predictors of the most severe measure of energy insecurity and that race, health and housing conditions remain positive correlates of energy insecurity across all three measures, even when income is accounted for.

\section{The impact of COVID-19 on energy insecurity}

To this point, the analysis has provided suggestive evidence about the relationship between the early period of the COVID-19 pandemic and energy insecurity by comparing the prevalence and determinants of energy insecurity at different points in time. To further examine this relationship, we analysed the responses to several COVID-19-related survey items: whether they had received a COVID-19 stimulus payment (part of the Coronavirus Aid, Relief and Economic Security Act), whether their employment status had changed due to the pandemic and whether someone in their household had symptoms of or a positive test for COVID-19. Lastly, we included a factor score (see Supplementary Table 3) to measure 'COVID-19 hardship' or the material hardship (for example, financial, food and medical insecurity) a respondent experienced in the immediate aftermath of the pandemic.

A larger proportion of households that were adversely affected by the public health crisis also experienced all three levels of energy insecurity in the past month (Fig. 4). Additionally, those that received a stimulus cheque were less likely to face issues as the pandemic began to unfold.

Next, we re-estimated the logistic regression model for the energy insecurity measures reported during the early COVID-19 period and included these variables as regressors. The full set of results (Supplementary Table 4) reveals that, in general, the same set of covariates that were correlated with energy insecurity in the past year remained statistically associated with energy insecurity in the past month, even when we controlled for the potential confounding effects of COVID-19.

Conditions associated with COVID-19 are positively associated with energy insecurity (Fig. 5). Material hardship and unemployment and/or lost hours due to the pandemic are statistically associated 


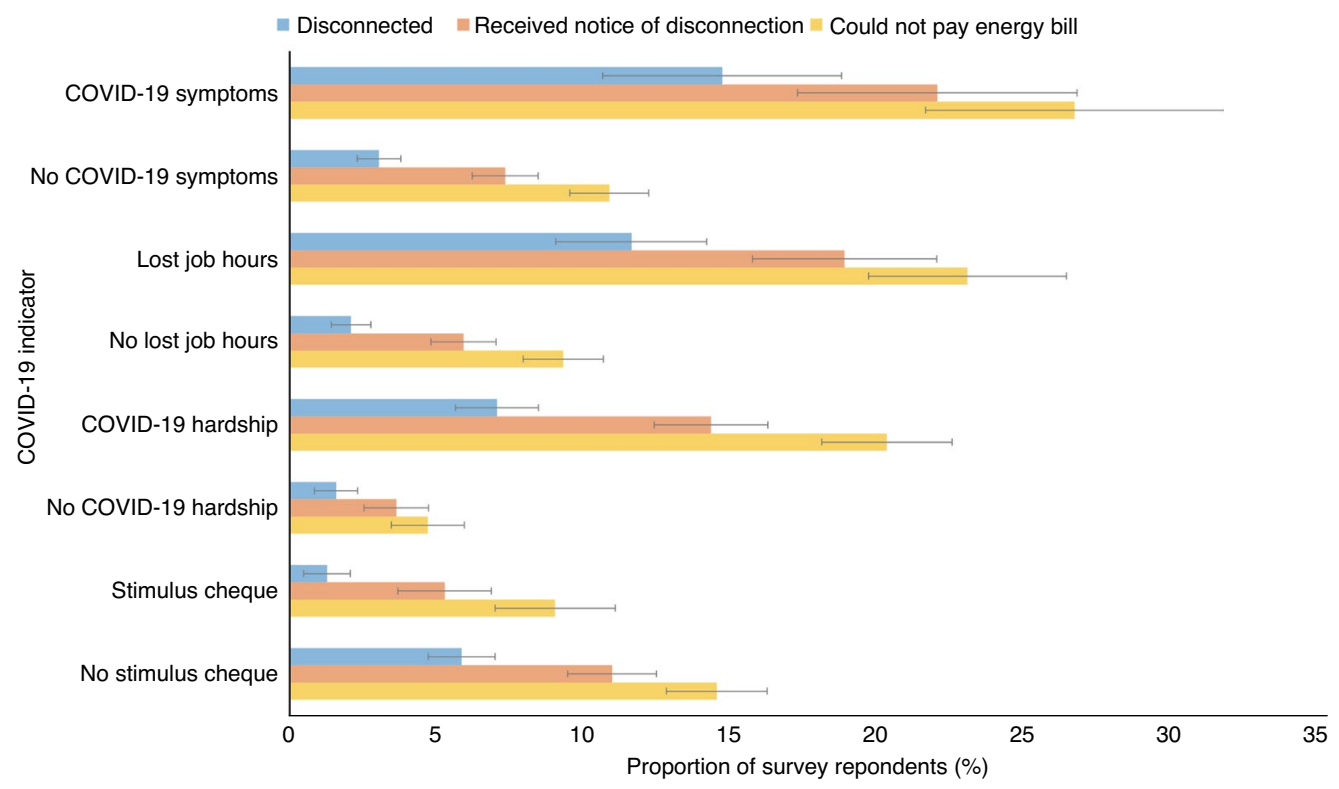

Fig. 4 | COVID-19-related conditions and energy insecurity. The proportion of households that could not afford an energy bill (yellow), received a disconnection notice (orange), or were disconnected from the grid (blue) in the past month by various COVID-19-related measures. Grey bars represent $95 \% \mathrm{Cls}(n=2,381)$.

with all three forms of energy insecurity. Those that reported having a household member with symptoms or a COVID-19 diagnosis had greater odds of reporting an inability to pay one's energy bill, whereas those who received a stimulus cheque had greater odds of avoiding utility disconnection. The results regarding the COVID-19 stimulus assistance may also capture unobserved characteristics about those that received this cash assistance (that is, stable residence and bank accounts). It is important to note that only about one-third of respondents reported that they had received a stimulus cheque.

\section{Addressing energy insecurity is imperative}

This study provides estimates of the prevalence of energy insecurity among low-income households in the United States, which reveal that approximately 4.8 million households were unable to pay at least one energy bill during the past year and a similar number received a notice from their utility that they faced the threat of disconnection from energy service. Of those that received a notice, more than two in five families had their energy shut off. These numbers equate to tens of millions of Americans.

We also found notable disparities across all three indicators of energy insecurity, which include race, families with young children, households with an individual who relies on an electronic medical device, and people who live in residences in poor condition. The latter results are consistent with previous works that identify housing conditions and inefficient housing stock as a leading contributor to energy insecurity in the United States ${ }^{18,34,39-41}$. It is important to emphasize, moreover, that these inequalities exist, especially between Black and Hispanic households and White households, even when income is accounted. Given that people who experience these circumstances also tend to suffer from other types of material hardship, such as food insecurity ${ }^{13,20}$ and limited access to affordable health care $^{18}$, these disparities in energy insecurity probably amplify the challenges that these vulnerable populations face.

Further, our analysis indicates that energy insecurity has intensified because of the COVID-19 pandemic. Both the economic dislocation and, to some extent, the virus itself are associated with difficulty in meeting household energy needs during the early period of the pandemic. Moreover, our results provide strong suggestive evidence that the sociodemographic disparities that existed prepandemic-that is, during 'normal' circumstances-widened during the early months of the crisis. This finding is consistent with past research that showed that economic downturns tend to exacerbate material hardship among already vulnerable populations ${ }^{42,43}$.

During the early months of the pandemic, US federal, state and local governments put a range of emergency measures in place to help households cope with material hardship (for example, eviction and utility shutoff moratoriums, expanded unemployment insurance, discounted housing payments and direct cash assistance). In the case of the electricity service specifically, many states adopted temporary shutoff protections to supplement the patchwork of existing state-level policies that limit the circumstances in which utilities can disconnect customers ${ }^{44}$. As these temporary measures elapse, one might anticipate further incidence and severity of energy insecurity. In the short term, additional financial assistance programmes, extensions of state-level protections and the establishment of a national moratorium on electricity disconnections would mitigate household energy insecurity through the duration of the pandemic. In the long term, potential government interventions might include investments in energy efficiency programmes to help households afford energy.

There remains much to learn about the prevalence and determinants of energy insecurity. The findings in this study about race and ethnicity require further inquiry. Past work emphasizes that Blacks and Hispanics disproportionately experience energy insecurity ${ }^{45}$; the analysis presented here indicates that this association holds, even after controlling for income and housing conditions. That is, there is something left unexplained about the energy experiences of Black and Hispanic households that needs to be identified to fully understand the prevalence of energy insecurity in these groups.

Among other unanswered questions are what strategies people employ to cope with energy insecurity. There are at least two important dimensions to examine. The first dimension relates to the financial strategies that people adopt to balance their energy needs with material hardship. Sociological research on material hardship, for example, found that households engage in credit balancing and strategic non-payment to manage their overall expenses ${ }^{46}$. This type 


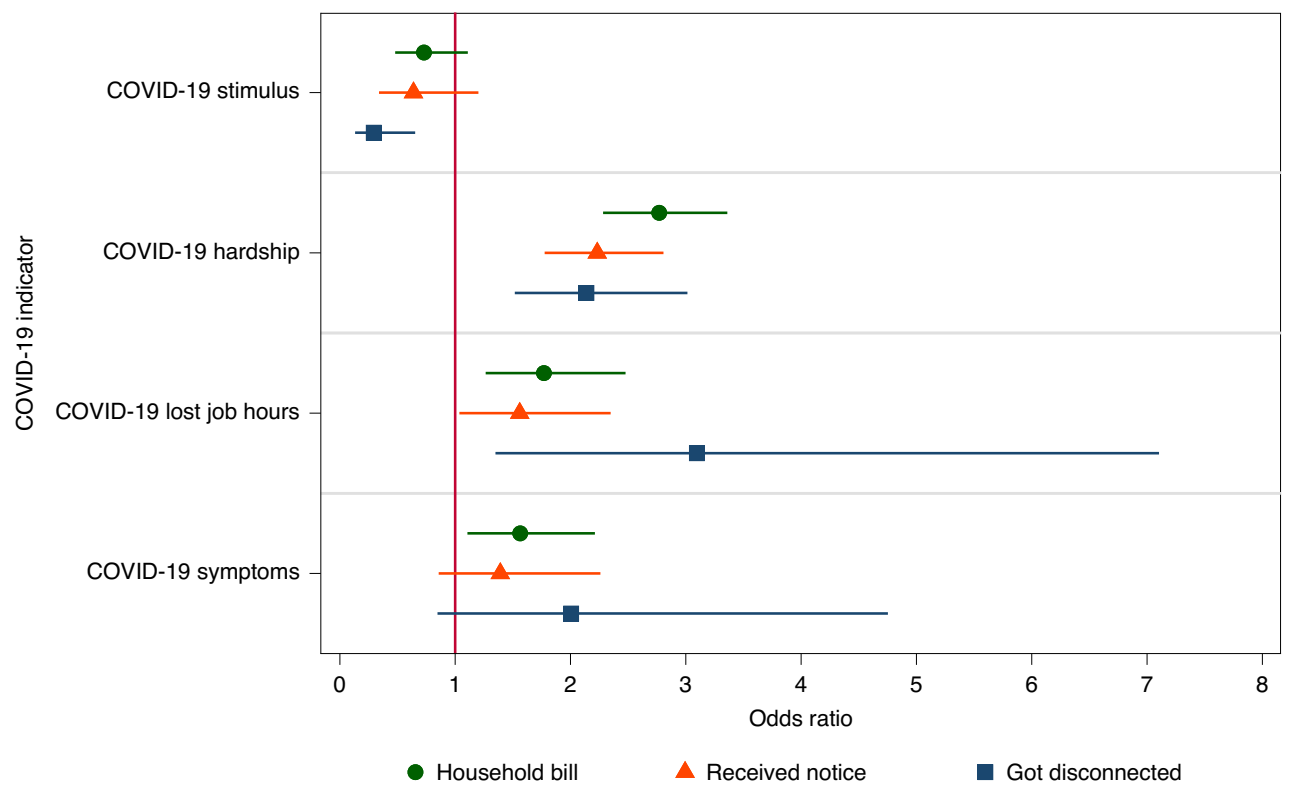

Fig. 5 | COVID-19-related correlates of energy insecurity. Odds ratios with 95\% Cls, estimated from logistic regression models ( $n=2,287)$. Models also include variables for households with children under 5 , household member with a disability, unemployed, household member using an electronic device, retired, Black, Hispanic, other race or ethnicity, gender, age, income, education, dwelling type (mobile home, apartment, attached home), housing conditions, percent zip code urban and receipt of government assistance. Full model estimates are provided in Supplementary Table 4. Robustness checks using ordinary least squares estimation and other variations defined in the Methods section are presented in Supplementary Tables 5-12.

of behaviour may result in households accruing long-term debts, which may turn a short-term problem into a longer-term one. Households may also seek out relief or financial assistance from both formal (for example, utilities and government programmes such as the Low Income Home Energy Assistance Program or the Weatherization Assistance Program) and informal sources (for example, family and friends, faith-based organizations and other non-profit organizations).

Beyond the financial aspects, scholars should also continue to examine the behavioural response of individuals to energy insecurity $^{1}$, by which we mean how people cope with less electricity consumption, either because of concerns about affordability or due to the loss of service as a result of non-payment. For example, little is known, at least from large, quantitative studies, about how people stay cool during the summer months or warm during the winter months if they cannot afford to turn on their air conditioning or heat. Research on behaviours during times of crisis suggests that individuals may do more risky things ${ }^{9,10}$, which in this context could lead to severe adverse effects to health and well-being.

\section{Methods}

Survey design and implementation. The survey was fielded in an online format through a contract with YouGov, a private polling and market research firm. YouGov generates representative samples using a matched sample methodology. YouGov develops a target population from general population studies, from which it draws a random set of respondents to create a target sample. Using a matching algorithm, the firm selects potential respondents from its US panel of approximately two million opt-in participants that match the target sample ${ }^{47}$, working with partner organizations to enhance its sample when necessary. YouGov's survey approach has been validated extensively ${ }^{47-49}$.

For this survey, YouGov interviewed a total of 2,914 respondents who were matched down to the final dataset of 2,381 participants, using a sampling frame on gender, age, race and education constructed by stratified sampling from the 2017 ACS one-year sample of individuals whose income is at or below $200 \%$ of the FPL. The matched cases were weighted to the sampling frame using propensity scores, and then the matched cases and the frame were combined and a logistic regression was estimated for inclusion in the frame. The propensity score function included age, gender, race and/or ethnicity, education and geographical region. The propensity scores were grouped into deciles of the estimated propensity score in the frame and poststratified according to these deciles. The weights were then poststratified on a four-way stratification of gender, age (four categories), race (four categories) and education (four categories) to produce the final weight, which was used in all the reported results and analyses. The survey has a margin of error of $2 \%$.

Our research design focused on low-income households because energy insecurity tends to be more heavily concentrated among households in this economic category $y^{50}$. We selected an income threshold of at or below $200 \%$ of the FPL for several reasons: (1) scholars ${ }^{51}$ and practitioners ${ }^{52}$ have previously relied on $200 \%$ of the FPL as an indicator of low-income households in the United States;

(2) federal energy assistance programmes set their income limits at $150 \%$ of the FPL, so surveying households at or below $200 \%$ of the FPL allows us to collect a sample of households that are within and above income eligibility thresholds, and (3) we were more likely to achieve a nationally representative sample of US households by sampling households at or below $200 \%$ of the FPL, rather than $100 \%$ of the FPL.

The survey was administered between 30 April and 25 May 2020, and took respondents, on average, ten minutes to complete. We asked survey respondents to answer a series of questions about their housing conditions, health status and energy insecurity over the past year, as well as over the past month (a period that we define in this study as during April or May 2020). We also asked a series of questions specifically about how respondents' circumstances had changed since the onset of the COVID-19 pandemic, which we defined as beginning in the second week of March 2020, which roughly coincides with the timing of when state governments began to enact stay-at-home orders and social distancing measures.

Statistical analysis. To measure the determinants of energy insecurity, we employed a series of logistic regressions. The binary dependent variables measure whether (1) a respondent's household could not pay their energy bill, (2) their household received a disconnection notice and (3) their household electricity was disconnected. The survey asked respondents about these outcomes for different time frames, which enabled us to compare energy insecurity over the course of the past year with that in the past month (during the economic crisis brought about by the pandemic). To control for potential omitted variable biases due to unobserved interstate (across state) variation, we included a series of state dummy variables, which allowed us to exploit within-state variation. We further clustered standard errors at the state level to account for within-cluster correlations. We also conducted the year, month and COVID-19 specific analyses using linear regression analysis (Supplementary Tables 5 and 6). As for the logistic regressions, we included state dummy variables and clustered our standard errors at the state level. We found consistent outcomes across both the logistic and linear empirical specification for all three of our models.

Lastly, we conducted a final set of robustness checks that employed both the logistic and linear regression empirical specifications. To test whether our 
COVID-19 measures were accounting for unmeasured variation, we narrowed the sample to those who experienced energy insecurity in the past year but not in the past month. This allowed us to check whether the material hardship, economic and health factors that relate to the pandemic remain statistically linked to energy insecurity. If the factors remained correlated, it suggests that the COVID-19 measures were merely accounting for some omitted variable and were not specific contributors to energy insecurity in the past month, or as the public health crisis began to unfold. Both the logistic (Supplementary Table 7) and linear (Supplementary Table 8) models largely revealed a loss of significance across the COVID-19 measures, which indicates that the pandemic and the economic disruption it caused is, in fact, responsible for deepening energy insecurity in the past month across our survey population. We also tested a set of regressions with an additional control variable for respondents' self-reported average monthly energy bill expenditure. Although household energy expenditures is a positive and significant predictor of our measures of energy insecurity in both time periods, we did not include this as a variable in our main models because 110 survey respondents did not report this value. Therefore, the missing observations would reduce our sample size by roughly 5 percent. Importantly, when controlling for energy expenditures, our results remain relatively consistent for both the logistic (Supplementary Tables 9 and 10) and linear (Supplementary Tables 11 and 12) models.

Ethics. This research involved human subjects. It was approved by the Indiana University Office of Research Compliance, under protocol number 2004296209. In accordance with this protocol, informed consent was provided by all the study participants.

Reporting Summary. Further information on research design is available in the Nature Research Reporting Summary linked to this article.

\section{Data availability}

Data and associated materials used in this study are available in the Harvard Dataverse repository ${ }^{53}$, which includes the original survey data and codebook, the STATA data-processing do-file and the STATA data analysis do-file. Replication sources can be found at https://dataverse.harvard.edu/dataset. xhtml?persistentId=doi:10.7910/DVN/OMJWNB

Received: 27 August 2020; Accepted: 8 December 2020; Published online: 18 January 2021

\section{References}

1. Hernández, D. Understanding 'energy insecurity' and why it matters to health. Soc. Sci. Med. 167, 1-10 (2016).

2. Hernández, D., Aratani, Y. \& Jiang, Y. (2014). Energy Insecurity among Families with Children (National Center for Children in Poverty, 2014).

3. Residential Energy Consumption Survey 2015) (US Energy Information Administration, 2015).

4. Thomson, H., Bouzarovski, S. \& Snell, C. Rethinking the measurement of energy poverty in Europe: a critical analysis of indicators and data. Indoor Built Environ. 26, 879-901 (2017).

5. Thomson, H. \& Snell, C. Quantifying the prevalence of fuel poverty across the European Union. Energy Policy 52, 563-572 (2013).

6. Bouzarovski, S. \& Petrova, S. A global perspective on domestic energy deprivation: overcoming the energy poverty-fuel poverty binary. Energy Res. Soc. Sci. 10, 31-40 (2015).

7. Bouzarovski, S. Energy poverty in the European Union: landscapes of vulnerability. Wiley Interdiscip. Rev. Energy Environ. 3, 276-289 (2014).

8. Ürge-Vorsatz, D. \& Herrero, S. T. Building synergies between climate change mitigation and energy poverty alleviation. Energy Policy 49, 83-90 (2012).

9. Bednar, D. J. \& Reames, T. G. Recognition of and response to energy poverty in the United States. Nat. Energy 5, 432-439 (2020).

10. Meder, B., Le Lec, F. \& Osman, M. Decision making in uncertain times: what can cognitive and decision sciences say about or learn from economic crises? Trends Cogn. Sci. 17, 257-260 (2013).

11. Levy, R. \& Sledge, J. A Complex Portrait: An Examination of Small-Dollar Credit Consumers (Center for Financial Services Innovation, 2012).

12. Campbell, R. B. Home Fires Involving Heating Equipment (National Fire Protection Association, 2016).

13. Bhattacharya, J., DeLeire, T., Haider, S. \& Currie, J. Heat or eat? Cold-weather shocks and nutrition in poor American families. Am. J. Public Health 93, 1149-1154 (2003).

14. Bohr, J. \& McCreery, A. C. Do energy burdens contribute to economic poverty in the United States? A panel analysis. Soc. Forces 99, 155-177 (2020).

15. Liddell, C. \& Morris, C. Fuel poverty and human health: a review of recent evidence. Energy Policy 38, 2987-2997 (2010).

16. Mendell, M. J., Mirer, A. G., Cheung, K., Tong, M. \& Douwes, J. Respiratory and allergic health effects of dampness, mold, and dampness-related agents: a review of the epidemiologic evidence. Environ. Health Perspect. 119, 748-756 (2011).
17. Shenassa, E. D., Daskalakis, C., Liebhaber, A., Braubach, M. \& Brown, M. Dampness and mold in the home and depression: an examination of mold-related illness and perceived control of one's home as possible depression pathways. Am. J. Public Health 97, 1893-1899 (2007).

18. Hernández, D. \& Siegel, E. Energy insecurity and its ill health effects: a community perspective on the energy-health nexus in New York City. Energy Res. Soc. Sci. 47, 78-83 (2019).

19. Berko, J., Ingram, D. D., Saha, S. \& Parker, J. D. Deaths Attributed to Heat, Cold, and Other Weather Events in the United States, 2006-2010 Report 76 (National Center for Health Statistics, Centers for Disease Control and Prevention, US Department of Health and Human Services, 2014).

20. Cook, J. T. et al. A brief indicator of household energy security: associations with food security, child health, and child development in US infants and toddlers. Pediatrics 122, e867-e875 (2008).

21. Frank, D. A. et al. Heat or eat: the Low Income Home Energy Assistance Program and nutritional and health risks among children less than 3 years of age. Pediatrics 118, e1293-e1302 (2006).

22. Nord, M. \& Kantor, L. S. Seasonal variation in food insecurity is associated with heating and cooling costs among low-income elderly Americans. J. Nutr. 136, 2939-2944 (2006).

23. Snyder, L. P. \& Baker, C. A. Affordable home energy and health: making the connections. AARP Public Policy Inst. 1, 5-36 (2010).

24. Garg, S. et al. Hospitalization rates and characteristics of patients hospitalized with laboratory-confirmed coronavirus disease 2019-COVID-NET, 14 States, March 1-30, 2020. Morbid. Mortal Wkly Rep. 69, 458-464 (2020).

25. Thebault, R., Ba Tran, A., Williams, V. The coronavirus is infecting and killing black Americans at an alarmingly high rate. Washington Post (7 April 2020).

26. Oppel Jr, R., Gebeloff, R. Lai, K., Wright, W., Smith, M. (2020). The Fullest Look Yet at the Racial inequity of Coronavirus. New York Times (5 July 2020)

27. Collins, C., Landivar, L. C., Ruppanner, L. \& Scarborough, W. J. (2020) COVID-19 and the Gender Gap in Work Hours. Gend. Work Organ. https://doi.org/10.1111/gwao.12506 (2020).

28. Fairlie, R. W., Couch, K. \& Xu, H. The Impacts of COVID-19 on Minority Unemployment: First Evidence from April 2020 CPS Microdata IZA DP no. 13264 (IZA Institute of Labor Economics, 2020).

29. Montenovo, L. et al. Determinants of Disparities in COVID-19 Job Losses Working Paper 27132 (National Bureau of Economic Research, 2020).

30. Carley, S. \& Konisky, D. M. The justice and equity implications of the clean energy transition. Nat. Energy 5, 569-577 (2020).

31. Jenkins, K. E., Stephens, J. C., Reames, T. G. \& Hernández, D. Towards impactful energy justice research: transforming the power of academic engagement. Energy Res. Soc. Sci. 67, 101510 (2020).

32. Hernández, D., Jiang, Y., Carrión, D., Phillips, D. \& Aratani, Y. Housing hardship and energy insecurity among native-born and immigrant low-income families with children in the United States. J. Child. Poverty 22 77-92 (2016).

33. Mohr, T. M. Fuel poverty in the US: evidence using the 2009 residential energy consumption survey. Energy Econ. 74, 360-369 (2018).

34. Reames, T. G. Targeting energy justice: exploring spatial, racial/ethnic and socioeconomic disparities in urban residential heating energy efficiency. Energy Policy 97, 549-558 (2016).

35. Lyubich, E. The Race Gap in Residential Energy Expenditures Energy Institute WP 306 (Energy Institute at Haas, 2020).

36. Jessel, S., Sawyer, S. \& Hernández, D. Energy, poverty, and health in climate change: a comprehensive review of an emerging literature. Front. Public Health 7, 357 (2019).

37. Graff, M. \& Carley, S. COVID-19 assistance needs to target energy insecurity. Nat. Energy 5, 352-354 (2020).

38. Gross Domestic Product, Fourth Quarter and Year 2019 (Advance Estimate) (Bureau of Economic Analysis, 2020).

39. Min, J., Hausfather, Z. \& Lin, Q. F. A high-resolution statistical model of residential energy end use characteristics for the United States. J. Ind. Ecol. 14, 791-807 (2010).

40. Dubois, U. From targeting to implementation: the role of identification of fuel poor households. Energy Policy 49, 107-115 (2012).

41. Santamouris, M. et al. On the relation between the energy and social characteristics of the residential sector. Energy Build. 39, 893-905 (2007)

42. Pilkauskas, N. V., Currie, J. M. \& Garfinkel, I. The great recession, public transfers, and material hardship. Soc. Serv. Rev. 86, 401-427 (2012).

43. Huang, J., Birkenmaier, J. \& Kim, Y. Job loss and unmet health care needs in the economic recession: different associations by family income. Am. J. Public Health 104, e178-e183 (2014)

44. Flaherty, M., Carley, S. \& Konisky, D. M. Electric utility disconnection policy and vulnerable populations. Electr. J. 33, 106859 (2020).

45. Lewis, J., Hernández, D. \& Geronimus, A. Energy efficiency as energy justice: addressing racial inequities through investments in people and places. Energ. Effic. 13, 419-423 (2020). 
46. Seefeldt, K. S. Constant consumption smoothing, limited investments, and few repayments: the role of debt in the financial lives of economically vulnerable families. Soc. Serv. Rev. 89, 263-300 (2015).

47. Vavreck, L. \& Rivers, D. The 2006 cooperative congressional election study. J. Elect. Public Opin. Parties 18, 355-366 (2008).

48. Ansolabehere, S. \& Rivers, D. Cooperative survey research. Annu. Rev. Political Sci. 16, 307-329 (2013).

49. Ansolabehere, S. \& Schaffner, B. F. Does survey mode still matter? Findings from a 2010 multi-mode comparison. Political Anal. 22, 285-303 (2014).

50. Ross, L., Drehobl, A. \& Stickles, B. The High Cost of Energy in Rural America: Household Energy Burdens and Opportunities for Energy Efficiency (American Council for an Energy Efficient Economy, 2018).

51. She, P. \& Livermore, G. A. Material hardship, poverty, and disability among working-age adults. Soc. Sci. Q. 88, 970-989 (2007).

52. Karpman, M., Gonzalez, D., Zuckerman, S. \& Adams, G. What Explains the Widespread Material Hardship among Low-Income Families with Children? (Urban Institute, 2018).

53. Memmott, T., Konisky, D., Carley, S. \& Graff, M. Replication Data for: Sociodemographic Disparities in Energy Insecurity among Low-Income Households Before and During the COVID-19 Pandemic (Harvard Dataverse, 2020); https://doi.org/10.7910/DVN/OMJWNB

54. Distribution of Total Population by Federal Poverty Level (Kaiser Family Foundation, 2018)

\section{Acknowledgements}

We thank D. Hernández for sharing her survey instrument on energy insecurity, which informed our own survey design. This research was supported through funding provided by the National Science Foundation, the Alfred P. Sloan Foundation, the Environmental Resilience Institute, funded by Indiana University's Prepared for Environmental Change Grand Challenge initiative, and the Indiana University's Office of the Vice President of Research.

\section{Author contributions}

All the authors contributed to the conceptualization, research design, analysis and writing of this article.

\section{Competing interests}

The authors declare no competing interests.

\section{Additional information}

Supplementary information is available for this paper at https://doi.org/10.1038/ s41560-020-00763-9.

Correspondence and requests for materials should be addressed to S.C.

Peer review information Nature Energy thanks Stefan Bouzarovski, Lee White and the other, anonymous, reviewer(s) for their contribution to the peer review of this work.

Reprints and permissions information is available at www.nature.com/reprints. Publisher's note Springer Nature remains neutral with regard to jurisdictional claims in published maps and institutional affiliations.

(c) The Author(s), under exclusive licence to Springer Nature Limited 2021 


\section{Reporting Summary}

Nature Research wishes to improve the reproducibility of the work that we publish. This form provides structure for consistency and transparency in reporting. For further information on Nature Research policies, see our Editorial Policies and the Editorial Policy Checklist.

\section{Statistics}

For all statistical analyses, confirm that the following items are present in the figure legend, table legend, main text, or Methods section.

n/a Confirmed

$\bigotimes$ The exact sample size $(n)$ for each experimental group/condition, given as a discrete number and unit of measurement

$\triangle$ A statement on whether measurements were taken from distinct samples or whether the same sample was measured repeatedly

The statistical test(s) used AND whether they are one- or two-sided

Only common tests should be described solely by name; describe more complex techniques in the Methods section.

$\bigotimes$ A description of all covariates tested

$\square$ A description of any assumptions or corrections, such as tests of normality and adjustment for multiple comparisons

$\checkmark$ A full description of the statistical parameters including central tendency (e.g. means) or other basic estimates (e.g. regression coefficient)

AND variation (e.g. standard deviation) or associated estimates of uncertainty (e.g. confidence intervals)

$\varnothing$ For null hypothesis testing, the test statistic (e.g. $F, t, r$ ) with confidence intervals, effect sizes, degrees of freedom and $P$ value noted Give $P$ values as exact values whenever suitable.

Х $\square$ For Bayesian analysis, information on the choice of priors and Markov chain Monte Carlo settings

Х $\square$ For hierarchical and complex designs, identification of the appropriate level for tests and full reporting of outcomes

$\bigotimes \square$ Estimates of effect sizes (e.g. Cohen's $d$, Pearson's $r$ ), indicating how they were calculated

Our web collection on statistics for biologists contains articles on many of the points above.

\section{Software and code}

Policy information about availability of computer code

Data collection All data collection is open source and can be found at: https://dataverse.harvard.edu/dataset.xhtml?persistentld=doi:10.7910/DVN/OMJWNB

Data analysis All data analysis is open source and can be found at: https://dataverse.harvard.edu/dataset.xhtml?persistentld=doi:10.7910/DVN/OMJWNB

For manuscripts utilizing custom algorithms or software that are central to the research but not yet described in published literature, software must be made available to editors and

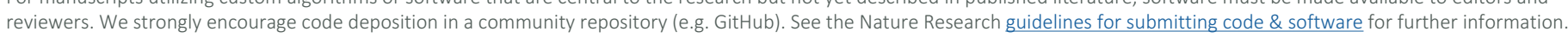

\section{Data}

Policy information about availability of data

All manuscripts must include a data availability statement. This statement should provide the following information, where applicable:

- Accession codes, unique identifiers, or web links for publicly available datasets

- A list of figures that have associated raw data

- A description of any restrictions on data availability

Data and associated materials used in this study are available in the Harvard Dataverse repository, including the original survey data and codebook, the STATA data processing do-file, and the STATA data analysis do-file. Replication sources can be found at: https://dataverse.harvard.edu/dataset.xhtml?persistentld=doi:10.7910/ DVN/OMJWNB 


\section{Field-specific reporting}

Please select the one below that is the best fit for your research. If you are not sure, read the appropriate sections before making your selection.

Life sciences $\quad$ Behavioural \& social sciences Ecological, evolutionary \& environmental sciences

For a reference copy of the document with all sections, see nature.com/documents/nr-reporting-summary-flat.pdf

\section{Behavioural \& social sciences study design}

All studies must disclose on these points even when the disclosure is negative.

Study description A quantitative cross-sectional study of energy insecurity before and during the COVID-19 pandemic

Research sample A nationally representative sample of 2,381 respondents at or below $200 \%$ of the Federal Poverty Line.

Sampling strategy The survey was fielded through YouGov, which generates representative samples using a matched sample methodology. YouGov develops a target population from general population studies, from which it draws a random set of respondents to create a target sample.

Data collection

The survey was fielded in an online format through a contract with YouGov.

Timing

The survey was administered between April 30 and May 25, 2020.

Data exclusions

No data were excluded.

Non-participation

No participants dropped out/declined participation.

Randomization

N/A

\section{Reporting for specific materials, systems and methods}

We require information from authors about some types of materials, experimental systems and methods used in many studies. Here, indicate whether each material, system or method listed is relevant to your study. If you are not sure if a list item applies to your research, read the appropriate section before selecting a response.

\begin{tabular}{l|lll}
\multicolumn{2}{l}{ Materials \& experimental systems } & & Methods \\
\hline n/a & Involved in the study & & \\
$\square / a$ & $\square$ Antibodies &
\end{tabular}

$\square \square$ Animals and other organisms

$\square \square$ Human research participants

$\square \square$ Clinical data

$\square \square$ Dual use research of concern

\section{Antibodies}

Antibodies used

Describe all antibodies used in the study; as applicable, provide supplier name, catalog number, clone name, and lot number.

Validation

\section{Eukaryotic cell lines}

Policy information about cell lines

Cell line source(s)

Authentication

Mycoplasma contamination
State the source of each cell line used.

Describe the authentication procedures for each cell line used OR declare that none of the cell lines used were authenticated.

Confirm that all cell lines tested negative for mycoplasma contamination OR describe the results of the testing for mycoplasma contamination OR declare that the cell lines were not tested for mycoplasma contamination. 


\section{Palaeontology and Archaeology}

Specimen provenance

Specimen deposition

Dating methods

Provide provenance information for specimens and describe permits that were obtained for the work (including the name of the issuing authority, the date of issue, and any identifying information).

Indicate where the specimens have been deposited to permit free access by other researchers.

If new dates are provided, describe how they were obtained (e.g. collection, storage, sample pretreatment and measurement), where they were obtained (i.e. lab name), the calibration program and the protocol for quality assurance OR state that no new dates are provided.

Tick this box to confirm that the raw and calibrated dates are available in the paper or in Supplementary Information.

Ethics oversight

Identify the organization(s) that approved or provided guidance on the study protocol, OR state that no ethical approval or guidance was required and explain why not.

Note that full information on the approval of the study protocol must also be provided in the manuscript.

\section{Animals and other organisms}

Policy information about studies involving animals; ARRIVE guidelines recommended for reporting animal research

Laboratory animals

Wild animals

Field-collected samples

Ethics oversight
For laboratory animals, report species, strain, sex and age OR state that the study did not involve laboratory animals.

Provide details on animals observed in or captured in the field; report species, sex and age where possible. Describe how animals were caught and transported and what happened to captive animals after the study (if killed, explain why and describe method; if released, say where and when) OR state that the study did not involve wild animals.

For laboratory work with field-collected samples, describe all relevant parameters such as housing, maintenance, temperature, photoperiod and end-of-experiment protocol OR state that the study did not involve samples collected from the field.

Identify the organization(s) that approved or provided guidance on the study protocol, OR state that no ethical approval or guidance was required and explain why not.

Note that full information on the approval of the study protocol must also be provided in the manuscript.

\section{Human research participants}

Policy information about studies involving human research participants

Population characteristics

Describe the covariate-relevant population characteristics of the human research participants (e.g. age, gender, genotypic information, past and current diagnosis and treatment categories). If you filled out the behavioural \& social sciences study design questions and have nothing to add here, write "See above."

Recruitment

Describe how participants were recruited. Outline any potential self-selection bias or other biases that may be present and how these are likely to impact results.

Ethics oversight

Identify the organization(s) that approved the study protocol.

Note that full information on the approval of the study protocol must also be provided in the manuscript.

\section{Clinical data}

Policy information about clinical studies

All manuscripts should comply with the ICMJE guidelines for publication of clinical research and a completed CONSORT checklist must be included with all submissions.

Clinical trial registration

Study protocol

Data collection

Outcomes
Provide the trial registration number from ClinicalTrials. gov or an equivalent agency.

Note where the full trial protocol can be accessed OR if not available, explain why.

Describe the settings and locales of data collection, noting the time periods of recruitment and data collection. 
Policy information about dual use research of concern

Hazards

Could the accidental, deliberate or reckless misuse of agents or technologies generated in the work, or the application of information presented in the manuscript, pose a threat to:
No $\mid$ Yes
$\square \square$ Public health
$\square \square$ National security
$\square \square$ Crops and/or livestock
$\square \square$ Ecosystems
$\square \square$ Any other significant area

\section{Experiments of concern}

Does the work involve any of these experiments of concern:
No $\mid$ Yes
$\square \square$ Demonstrate how to render a vaccine ineffective
$\square \square$ Confer resistance to therapeutically useful antibiotics or antiviral agents
$\square \square$ Enhance the virulence of a pathogen or render a nonpathogen virulent
$\square \square$ Increase transmissibility of a pathogen
$\square \square$ Alter the host range of a pathogen
$\square \square$ Enable evasion of diagnostic/detection modalities
$\square \square$ Enable the weaponization of a biological agent or toxin
$\square \square$ Any other potentially harmful combination of experiments and agents

ChIP-seq

\section{Data deposition}

$\square$ Confirm that both raw and final processed data have been deposited in a public database such as GEO.

$\square$ Confirm that you have deposited or provided access to graph files (e.g. BED files) for the called peaks.

Data access links

May remain private before publication.

Files in database submission

Genome browser session (e.g. $\underline{\operatorname{UCSC}})$

\section{Methodology}

Replicates

Sequencing depth

Antibodies

Peak calling parameters

Data quality

Software
For "Initial submission" or "Revised version" documents, provide reviewer access links. For your "Final submission" document, provide a link to the deposited data.

Provide a list of all files available in the database submission.

Provide a link to an anonymized genome browser session for "Initial submission" and "Revised version" documents only, to enable peer review. Write "no longer applicable" for "Final submission" documents. 
Plots

Confirm that:

$\square$ The axis labels state the marker and fluorochrome used (e.g. CD4-FITC).

$\square$ The axis scales are clearly visible. Include numbers along axes only for bottom left plot of group (a 'group' is an analysis of identical markers).

$\square$ All plots are contour plots with outliers or pseudocolor plots.

A numerical value for number of cells or percentage (with statistics) is provided.

\section{Methodology}

Sample preparation

Describe the sample preparation, detailing the biological source of the cells and any tissue processing steps used.

Instrument

Identify the instrument used for data collection, specifying make and model number.

Software

Describe the software used to collect and analyze the flow cytometry data. For custom code that has been deposited into a community repository, provide accession details.

Cell population abundance

Describe the abundance of the relevant cell populations within post-sort fractions, providing details on the purity of the samples and how it was determined.

Gating strategy

Describe the gating strategy used for all relevant experiments, specifying the preliminary FSC/SSC gates of the starting cell population, indicating where boundaries between "positive" and "negative" staining cell populations are defined.

Tick this box to confirm that a figure exemplifying the gating strategy is provided in the Supplementary Information.

\section{Magnetic resonance imaging}

\section{Experimental design}

Design type

Design specifications

Behavioral performance measures

\section{Acquisition}

Imaging type(s)

Field strength

Sequence \& imaging parameters

Area of acquisition

Diffusion MRI $\square$ Used

Preprocessing

Preprocessing software

Normalization

Normalization template

Noise and artifact removal
Indicate task or resting state; event-related or block design

Specify the number of blocks, trials or experimental units per session and/or subject, and specify the length of each trial or block (if trials are blocked) and interval between trials.

State number and/or type of variables recorded (e.g. correct button press, response time) and what statistics were used to establish that the subjects were performing the task as expected (e.g. mean, range, and/or standard deviation across subjects).

Specify: functional, structural, diffusion, perfusion.

Specify in Tesla

Specify the pulse sequence type (gradient echo, spin echo, etc.), imaging type (EPI, spiral, etc.), field of view, matrix size slice thickness, orientation and TE/TR/flip angle.

State whether a whole brain scan was used OR define the area of acquisition, describing how the region was determined. Not used
Provide detail on software version and revision number and on specific parameters (model/functions, brain extraction, segmentation, smoothing kernel size, etc.)

If data were normalized/standardized, describe the approach(es): specify linear or non-linear and define image types used for transformation OR indicate that data were not normalized and explain rationale for lack of normalization.

Describe the template used for normalization/transformation, specifying subject space or group standardized space (e.g original Talairach, MNI305, ICBM152) OR indicate that the data were not normalized.

Describe your procedure(s) for artifact and structured noise removal, specifying motion parameters, tissue signals and physiological signals (heart rate, respiration). 


\section{Statistical modeling \& inference}

Model type and settings

Effect(s) tested

Specify type of analysis:

Statistic type for inference (See Eklund et al. 2016)

Correction

Specify type (mass univariate, multivariate, RSA, predictive, etc.) and describe essential details of the model at the first and second levels (e.g. fixed, random or mixed effects; drift or auto-correlation).

Define precise effect in terms of the task or stimulus conditions instead of psychological concepts and indicate whether ANOVA or factorial designs were used.

\section{Whole brain $\square$ ROI-based $\square$ Both}

Specify voxel-wise or cluster-wise and report all relevant parameters for cluster-wise methods.

Describe the type of correction and how it is obtained for multiple comparisons (e.g. FWE, FDR, permutation or Monte Carlo).

Models \& analysis

\begin{tabular}{l|l} 
n/a Involved in the study \\
$\square$ Functional and/or effective connectivity \\
$\square$ Graph analysis \\
$\square$ Multivariate modeling or predictive analysis
\end{tabular}

Functional and/or effective connectivity

Graph analysis

Multivariate modeling and predictive analysis
Report the measures of dependence used and the model details (e.g. Pearson correlation, partial correlation, mutual information).

Report the dependent variable and connectivity measure, specifying weighted graph or binarized graph, subject-or group-level, and the global and/or node summaries used (e.g. clustering coefficient, efficiency, etc.).

Specify independent variables, features extraction and dimension reduction, model, training and evaluation metrics. 